\title{
WAVE ANALYSIS OF A L-BEAM STRUCTURE WITH A BLOCKING MASS
}

\author{
Johnny Tiu ${ }^{1 *}$ and Richard Bachoo ${ }^{2}$ \\ ${ }^{1,2}$ Faculty of Engineering, The University of the West Indies, Trinidad \\ ${ }^{1}$ Email: johnny.tiu@sta.uwi.edu*(Corresponding author) \\ 2Email: richard.bachoo@sta.uwi.edu
}

\begin{abstract}
The wave vibration approach regards the vibrations present within a structure as waves, whereby each wave flows along a structural member and upon meeting a discontinuity; portions of the incident wave are reflected and transmitted across the discontinuity. The reflected, transmitted and propagating wave transformations are represented mathematically by matrices, which are used to develop a set of wave relation equations at each discontinuity that can be used to describe the frequency response of the system holistically. This method creates a systematic approach of analysing structures by utilizing common cases as building blocks for a specific structure. The L-joint, described as two beams meeting at right angles; is a ubiquitous case for spatial portal and structural frames, which may become geometrically complex. Such structures are well suited to a wave vibration approach due to the large number of geometric changes and the prevalence as well as recurrence of specific cases. In this paper, the L-joint expanded to include a blocking mass, typically employed in structural systems and allows for the isolation and reflection of vibration away from contiguous structural elements. Included are; variance of transmission and reflection matrix components as the size of the blocking mass increases, numerical examples and comparison to a Finite Element Model developed in ANSYS.
\end{abstract}

Keywords: Blocking mass, L-joint, Wave vibration approach, Timoshenko beam.

https://doi.org/10.47412/EWGF2313

\section{Introduction}

The dynamic response of the structure is of great interest in several industries, as they may experience dynamic loading in the form of harmonic excitations from rotating machinery or random vibrations stemming from wind excitations or earthquakes. The response must be taken into consideration during the design process of any structure to determine whether the structure is appropriate for the given conditions and possible excitations otherwise the natural frequencies may be excited and the system will experience large displacements and stress and ultimately may result in catastrophic failure.

The modelling of structure can be undertaken using various methods; Finite Element Method (FEM) [39][47], Statistical Energy Analysis (SEA) [8][52] and wave propagation [29][31][32], each having their own inherent advantages and applications. These methods have varying suitability that hinges on the structure being modelled (in terms of material, dimensions and geometry) as well as the range of frequencies that are being investigated. Blocking masses have been investigated using SEA and FEM [50], and Wave analysis [14], with the former being applied to L-shaped plates and the latter to symmetric and eccentric blocking masses along a uniform wave guide.

Using the wave vibration approach, this paper models the free, in-plane, bending vibration of an L-beam structure and evaluates the suitability of this method as it applies to modelling blocking masses within the L-beam structure by comparing results of varying two parameters (blocking mass dimensions and material) 
to a Finite-Element Model developed in Ansys.

\section{Development of Wave Propagation Equations}

The means of developing the matrices used in the wave vibration approach is heavily based off of Mace [29] and Mei's method [31][32][33]. In particular, Mei [32] used the wave approach to model the L-beam structure with various boundary conditions. Consequently, the subsequent theoretical foundation presented in Sections 2 and 3 is based on Mei's [32] work.

\subsection{Equations of Motion}

From [20] the equations of motion of a beam, ignoring damping for a bending and longitudinal vibration are:

$$
\begin{gathered}
\text { GAK }\left[\frac{\partial \psi(x, t)}{\partial t}-\frac{\partial^{2} y(x, t)}{\partial x^{2}}\right]+\rho A \frac{\partial^{2} y(x, t)}{\partial t^{2}}=q(x, t) \\
E I \frac{\partial^{2} \Psi(x, t)}{\partial x^{2}}+G A \kappa\left[\frac{\partial y(x, t)}{\partial x}-\Psi(x, t)\right]-\rho I \frac{\partial^{2} \Psi(x, t)}{\partial t^{2}}=0 \\
\rho A \frac{\partial^{2} \mathbf{u}(x, t)}{\partial t^{2}}-E A \frac{\partial^{2} u(x, t)}{\partial x^{2}}=p(x, t)
\end{gathered}
$$

Where the material properties G, E, $\rho, \kappa$ are the shear modulus and Young's modulus, mass density and shear coefficient respectively. The geometric parameters; A, I, are the cross-sectional area and second moment of inertia respectively. Where $\mathrm{y}(\mathrm{x}, \mathrm{t}), \mathrm{u}(\mathrm{x}, \mathrm{t}), \psi(\mathrm{x}, \mathrm{t})$ are the deflections in the transverse, longitudinal and slope due to bending respectively each as functions of $\mathrm{x} \& \mathrm{t}$, the position along the $\mathrm{x}$-axis of the beam and time. $\mathrm{q}(\mathrm{x}, \mathrm{t})$ and $\mathrm{p}(\mathrm{x}, \mathrm{t})$ are externally applied loads in the transverse and longitudinal directions respectively. $\frac{\partial^{n}}{\partial^{n} \mathrm{x}}$ and $\frac{\partial^{n}}{\partial^{n} \mathrm{t}}$ refer to the $\mathrm{n}^{\text {th }}$ derivative of a function with respect to $\mathrm{x}$ and $\mathrm{t}$ respectively.

By assuming the beam undergoes harmonic motion under no external load, the solution takes the form of:

$$
u, y, \Psi=u_{0} e^{-i k x+i t \omega}, y_{0} e^{-i k x+i t \omega}, \Psi_{0} e^{-i k x+i t \omega}
$$

Eq. (1a) and Eq. (1b) can be represented temporally and spatially independent as:

$$
\left[\begin{array}{cc}
G A k^{2} \kappa-\rho A \omega^{2} & -i G A k \kappa \\
-i G A k \kappa & -E I k^{2}-G A \kappa+\rho I \omega^{2}
\end{array}\right]\left[\begin{array}{l}
y \\
\psi
\end{array}\right]=0
$$

The determinant of which gives two pairs of solutions for $\mathrm{k}_{1}$ and $\mathrm{k}_{2}$, the bending wavenumbers.

$$
k_{1}= \pm \sqrt{\frac{\rho \omega^{2}\left(\frac{1}{E}+\frac{1}{G \kappa}\right)+\frac{\sqrt{\rho} \omega \sqrt{4 A E G^{2} \kappa^{2}+I(E-G \kappa)^{2} \rho \omega^{2}}}{E G \sqrt{I} \kappa}}{2}}
$$

And

$$
k_{2}= \pm \sqrt{\frac{\rho \omega^{2}\left(\frac{1}{E}+\frac{1}{G \kappa}\right)-\frac{\sqrt{\rho} \omega \sqrt{4 A E G^{2} \kappa^{2}+I(E-G \kappa)^{2} \rho \omega^{2}}}{E G \sqrt{I} \kappa}}{2}}
$$


From Eq. (3) the amplitudes of $y(x)$ and $\psi(x)$ are coupled and can be represented in terms of each other.

$$
\begin{gathered}
\frac{\Psi}{y}=i \frac{\rho A \omega^{2}-k^{2} G A \kappa}{k G A \kappa} \\
y(x)=a_{1}^{+} e^{-i k_{1} x}+a_{2}^{+} e^{-k_{2} x}+a_{1}^{-} e^{i k_{1} x}+a_{2}^{-} e^{k_{2} x} \\
\psi(x)=b_{1}^{+} e^{-i k_{1} x}+b_{2}^{+} e^{-k_{2} x}+b_{1}^{-} e^{i k_{1} x}+b_{2}^{-} e^{k_{2} x} \\
\frac{b_{1}^{+}}{a_{1}^{+}}=-i P, \quad \frac{b_{1}^{-}}{a_{1}^{-}}=i P, \quad \frac{b_{2}^{+}}{a_{2}^{+}}=-N, \quad \frac{b_{2}^{-}}{a_{2}^{-}}=N \\
P=k_{1}\left(1-\frac{\omega^{2}}{k_{1}^{2} C_{s}^{2}}\right) \quad N=k_{2}\left(1+\frac{\omega^{2}}{k_{2}^{2} C_{s}^{2}}\right)
\end{gathered}
$$

By inspection of Eq. (1c) the longitudinal vibration is uncoupled from the bending waves and thus takes the form

$$
\begin{gathered}
\mathrm{AEk}^{2}-\mathrm{A} \rho \omega^{2}=0 \\
k_{3}= \pm \sqrt{\frac{\rho}{E}} \omega
\end{gathered}
$$

\subsection{Reflection at Supports}

The three commonly used classical boundary conditions; free, clamped and simply supported can be represented by a singular arbitrary boundary condition as depicted in Fig. 1. This general boundary condition is given by a translational spring, $\mathrm{K}_{\mathrm{T}}$ and rotational spring, $\mathrm{K}_{\mathrm{R}}$.

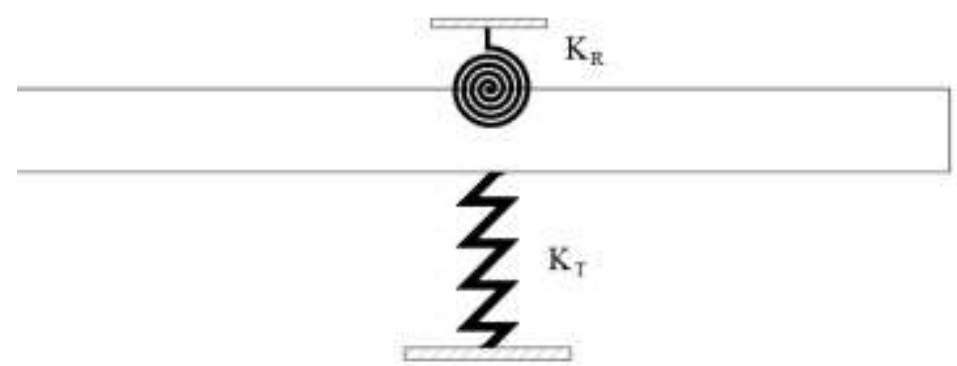

Figure 8: Generalised boundary condition

The generalised reflection matrix can be determined by using static equilibrium in the transverse and longitudinal direction as well as the balance of moments at the point using Eqn. 11.

$$
V=G A \kappa\left(\frac{\partial y}{\partial x}-\psi\right) M=E I \frac{\partial \psi}{\partial x} F=E A \frac{\partial u}{\partial x}
$$

Each specific boundary condition can then be found by applying the limit of $\mathrm{K}_{\mathrm{T}}$ and $\mathrm{K}_{\mathrm{R}}$ to either infinity or zero where appropriate [32]. 


$$
\begin{gathered}
\mathrm{r}_{c}=\left[\begin{array}{ccc}
-\frac{N+i P}{N-i P} & -\frac{2 N}{N-i P} & 0 \\
\frac{2 i P}{N-i P} & \frac{N+i P}{N-i P} & 0 \\
0 & 0 & -1
\end{array}\right] \\
\mathrm{r}_{f}=\left[\begin{array}{ccc}
\mathrm{r}_{s}=\left[\begin{array}{ccc}
-1 & 0 & 0 \\
0 & -1 & 0 \\
0 & 0 & -1
\end{array}\right] ; \\
\frac{\mathrm{k}_{1} \mathrm{k}_{2} N+i \mathrm{k}_{1} \mathrm{k}_{2} P-i \mathrm{k}_{1} N P-\mathrm{k}_{2} N P}{\mathrm{k}_{2} N-i \mathrm{k}_{1} \mathrm{k}_{2} P+i \mathrm{k}_{1} N P-\mathrm{k}_{2} N P} & -\frac{2 \mathrm{k}_{2}\left(\mathrm{k}_{2}-N\right) N}{i \mathrm{k}_{2} N P+\mathrm{k}_{1}\left(-i \mathrm{k}_{2} N-\mathrm{k}_{2} P+N P\right)} & 0 \\
\frac{2 \mathrm{k}_{1}(\mathrm{k} 1-P) P}{-\mathrm{k}_{2} N P+\mathrm{k}_{1}\left(\mathrm{k}_{2}(N-i P)+i N P\right)} & \frac{-\mathrm{k}_{2} N P+\mathrm{k}_{1}\left(\mathrm{k}_{2}(N+i P)-i N P\right)}{\mathrm{k}_{2} N P-\mathrm{k}_{1}\left(\mathrm{k}_{2}(N-i P)+i N P\right)} & 0 \\
0 & 0 & 1
\end{array}\right]
\end{gathered}
$$

\subsection{Propagation}

In the absence of any discontinuities (geometric, point supports, boundaries or excitations) as seen in Fig.

2 , the wave relation between two points along the beam of length, $x$ is given by the field transfer matrix $f(\mathrm{x})$ seen in Eqn. 14 .

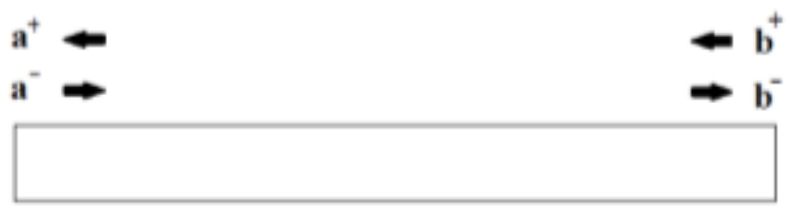

Figure 9: Wave reflection and transmission

$$
\begin{aligned}
b^{-} & =f(x) a^{-} ; \quad a^{+}=f(x) b^{+} \\
f(x) & =\left[\begin{array}{ccc}
e^{-i k_{1} x} & 0 & 0 \\
0 & e^{-k_{2} x} & 0 \\
0 & 0 & e^{-i k_{3} x}
\end{array}\right]
\end{aligned}
$$

\subsection{Reflection and Transmission Across L-joint}

The equilibrium conditions for the L-joint where i denotes the beam in reference, are given by [32]:

$$
\begin{gathered}
V_{i}=G A \kappa\left[\frac{\partial y_{i}}{\partial x_{i}}-\psi_{i}\right] \\
M_{i}=E I \frac{\partial y_{i}}{\partial x_{i}} \\
F_{i}=E A \frac{\partial u_{i}}{\partial x_{i}}
\end{gathered}
$$

The continuity conditions for the L-joint are given by [32]:

$$
\begin{gathered}
u_{1}=u_{J}, \quad u_{2}=y_{J} \\
y_{1}=y_{J}-\frac{h_{2}}{2} \psi_{J}, \quad y_{2}=-u_{J}+\frac{h_{1}}{2} \psi_{J} \\
\psi_{1}=\psi_{J}, \quad \Psi_{2}=\Psi_{J}
\end{gathered}
$$




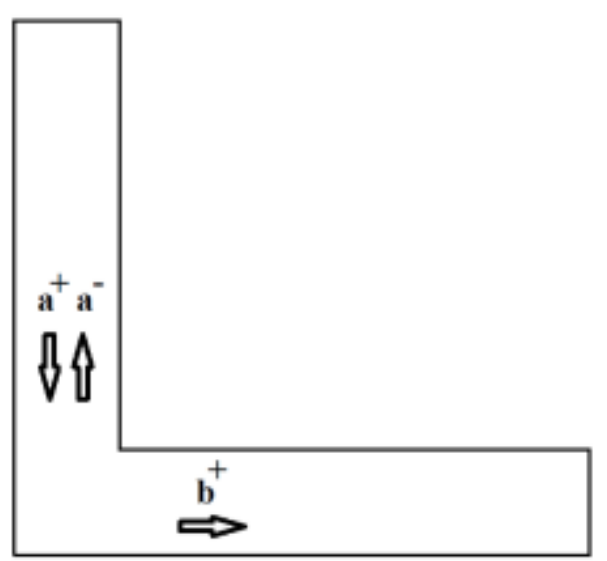

Figure 10: Reflection and transmission across a L-joint

$$
\mathrm{b}^{+}=\mathbf{t}_{\mathbf{j}} \mathrm{a}^{+}, \quad \mathrm{a}^{-}=\mathbf{r}_{\mathbf{j}} \mathrm{a}^{+}
$$

Where $\mathbf{t}_{\mathbf{j}}$ and $\mathbf{r}_{\mathbf{j}}$ are the transmission and reflection matrices across the joint.

In reference to beam 1 :

The time independents solutions to Eq. (15) are given as:

$$
\begin{aligned}
& y_{1}=a_{1}^{+} e^{-i k_{1} x_{1}}+a_{2}^{+} e^{-k_{2} x_{1}}+a_{1}^{-} e^{i k_{1} x_{1}}+a_{2}^{-} e^{k_{2} x_{1}} \\
& u_{1}=c^{+} e^{-i k_{3} x_{1}}+c^{-} e^{i k_{3} x_{1}} \\
& \psi_{1}=-i P a_{1}^{+} e^{-i k_{1} x_{1}}-N a_{2}^{+} e^{-k_{2} x_{1}}+i P a_{1}^{-} e^{i k_{1} x_{1}}+N a_{2}^{-} e^{k_{2} x_{1}} \\
& y_{2}=b^{+} e^{-i k_{1} x_{2}}+b_{N}^{+} e^{-k_{2} x_{2}} \\
& u_{2}=d^{+} e^{-i k_{3} x_{2}} \\
& \psi_{2}=-i P b^{+} e^{-i k_{1} x_{2}}-N b_{N}^{+} e^{-k_{2} x_{2}}
\end{aligned}
$$

From equations Eqs. (15), (17), (18) the continuity conditions are represented by:

$$
V_{1} a^{+}=V_{2} b^{+}+V_{3} a^{-}
$$

From equations Eqs. (16), (17), (18) the equilibrium conditions are represented by:

$$
V_{4} a^{+}=V_{5} b^{+}+V_{6} a^{+}
$$

Where $\mathrm{V}_{1-6}$ represent the respective coefficient matrices for each wave component

By using equations Eqs. (19-20) one can obtain the transmission matrix from beam 1 to 2 , $\mathrm{t}_{\mathrm{j} 12}$ and the reflection matrix of beam $1, \mathrm{r}_{\mathrm{j} 11}$.

Similarly, the transmission and reflection matrix for beam 2 can be found using the same method. 


\section{Analytical Model of L-beam}

This section takes focus on a simple L-beam structure whereby two beam elements are connected by an Ljoint. The system in Fig. 4 can be described using eight wave relation equations; two boundary reflection equations, two joint balance equations and four wave propagation equations.

\subsection{Wave Relation Equations}

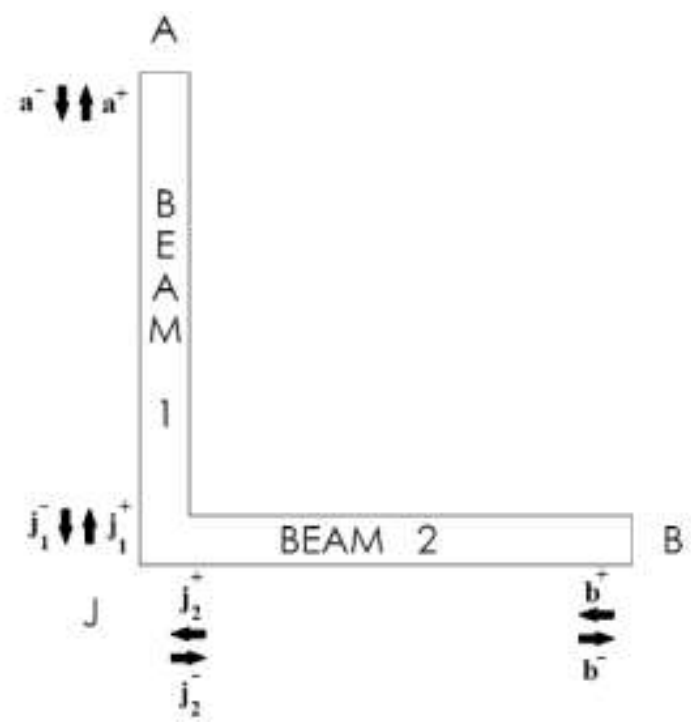

Figure 11: L-beam structure

At boundaries A and B respectively:

Across joint, J:

$$
\mathrm{a}^{-}=\mathrm{r}_{\mathrm{A}} \mathrm{a}^{+} ; \quad \mathrm{b}^{+}=\mathrm{r}_{\mathrm{B}} \mathrm{b}^{-}
$$

$$
\mathrm{j}_{2}^{-}=\mathrm{r}_{\mathrm{j} 22} \mathrm{j}_{2}^{+},+\mathrm{t}_{\mathrm{j} 12} \mathrm{j}_{1}^{-} ; \quad \mathrm{j}_{1}^{+}=\mathrm{r}_{\mathrm{j} 11} \mathrm{j}_{1}^{-}+\mathrm{t}_{\mathrm{j} 21 \mathrm{j}_{2}^{+}}
$$

Along beam 1 and beam 2 respectively:

$$
\begin{array}{ll}
\mathrm{j}_{1}^{-}=\mathrm{f}(x) \mathrm{a}^{-} ; & \mathrm{a}^{+}=\mathrm{f}(x) \mathrm{j}_{1}^{+} \\
\mathrm{b}^{-}=\mathrm{f}(x) \mathrm{j}_{2}^{-} ; & \mathrm{j}_{2}^{+}=\mathrm{f}(x) \mathrm{b}^{+}
\end{array}
$$

\subsection{Analytical Solutions}

As described by Mei [32] the analytical solutions to the natural frequencies can be found by expressing the wave relation equations as a product of the wave coefficient matrix (24 x 24), A and the wave component vector $(24 \times 1), Z$, setting the determinant of A to zero and obtaining the characteristic polynomial. The natural frequencies of the system can be obtained by determining the roots of the characteristic polynomial, this can be easily visualised by plotting the modulus of the characteristic polynomial and determine the cross points on the x-axis. Eq. (24) gives a reduced $8 \times 8$ form of the wave coefficient and component equation, whereby each entry represents the respective $3 \times 3$ matrix. The wave component vector and the wave coefficient by extension may take any order for the respective wave components. 


$$
\left[\begin{array}{cccccccc}
\mathrm{I} & -\mathrm{r}_{a} & 0 & 0 & 0 & 0 & 0 & 0 \\
0 & \mathrm{I} & 0 & -f & 0 & 0 & 0 & 0 \\
-f & 0 & \mathrm{I} & 0 & 0 & 0 & 0 & 0 \\
0 & 0 & -\mathrm{r}_{\mathrm{j} 11} & \mathrm{I} & 0 & -\mathrm{t}_{\mathrm{j} 12} & 0 & 0 \\
0 & 0 & -\mathrm{t}_{\mathrm{j} 12} & 0 & \mathrm{I} & -\mathrm{r}_{\mathrm{j} 22} & 0 & 0 \\
0 & 0 & 0 & 0 & 0 & \mathrm{I} & 0 & -f \\
0 & 0 & 0 & 0 & -f & 0 & \mathrm{I} & 0 \\
0 & 0 & 0 & 0 & 0 & 0 & -\mathrm{r}_{b} & \mathrm{I}
\end{array}\right]\left[\begin{array}{c}
a^{-} \\
a^{+} \\
\mathrm{j}_{1}^{-} \\
\mathrm{j}_{1}^{+} \\
\mathrm{j}_{2}^{-} \\
\mathrm{j}_{2}^{+} \\
\mathrm{b}^{-} \\
\mathrm{b}^{+}
\end{array}\right]=0
$$

Alternatively, the natural frequencies can be obtained by consolidating all the wave relation equations into a single equation through substitution of each relation. This forms an expression of only one wave component, where the determinant of the coefficient matrix $(3 \times 3)$ can be calculated to obtain a similar polynomial. Similarly, the natural frequencies can be obtained from the roots of the absolute value of this polynomial. This method proves to be more computationally efficient compared the first method outlined as the size of the coefficient matrix influences the computation time of the determinant by a factor of $\mathrm{n}^{3}$.

$$
\left[r_{b} \cdot f \cdot r_{j 22} \cdot f+r_{b} \cdot f \cdot t_{j 12} \cdot f \cdot\left[I-r_{a} \cdot f \cdot r_{j 11} \cdot f\right]^{-1} \cdot r_{a} \cdot f \cdot t_{j 12} \cdot f-I\right] \cdot b^{+}=0
$$

Eq. (25) shows an example of the alternative solution for the polynomial, which is given by the absolute value for the determinant of the coefficient matrix to $\mathrm{b}^{+}$. It should be noted that the solution need not be in terms of $b^{+}$and can take the form of any of the eight wave components.

\subsection{Numerical Example}

As an example; using the system described in section 3.1 and the following material properties: Young's modulus, $\mathrm{E}=200 \mathrm{GPa}$, shear modulus, $\mathrm{G}=76.9 \mathrm{GPa}$, Poisson's ratio, $v=0.3$, mass density, $\rho=7900$ $\mathrm{kg} / \mathrm{m}^{3}$, shear coefficient, $\kappa=10 *(1+v) /(12+11 v)$. The physical dimensions for the structure: beam thickness, $\mathrm{b}=25 \mathrm{~mm}$, beam width, $\mathrm{w}=25 \mathrm{~mm}$, beam $1 \& 2$ length, $\mathrm{x}=1 \mathrm{~m}$ and the boundary conditions for $\mathrm{A}$ and $\mathrm{B}$ are clamped.

The characteristic polynomial plot as obtained from Eq. (24) is shown in Fig. 5 and the natural frequencies are obtained numerically by calculating the local minima at the respective cross points on the $\mathrm{x}$-axis.

Table 3: Natural frequencies from characteristic polynomial

\begin{tabular}{|c|c|}
\hline Mode Number & Natural Frequency $(\mathrm{Hz})$ \\
\hline 1 & 87.741 \\
\hline 2 & 128.58 \\
\hline 3 & 282.48 \\
\hline 4 & 349.16 \\
\hline 5 & 576.5 \\
\hline 6 & 627.47 \\
\hline 7 & 688.97 \\
\hline 8 & 730.31 \\
\hline
\end{tabular}




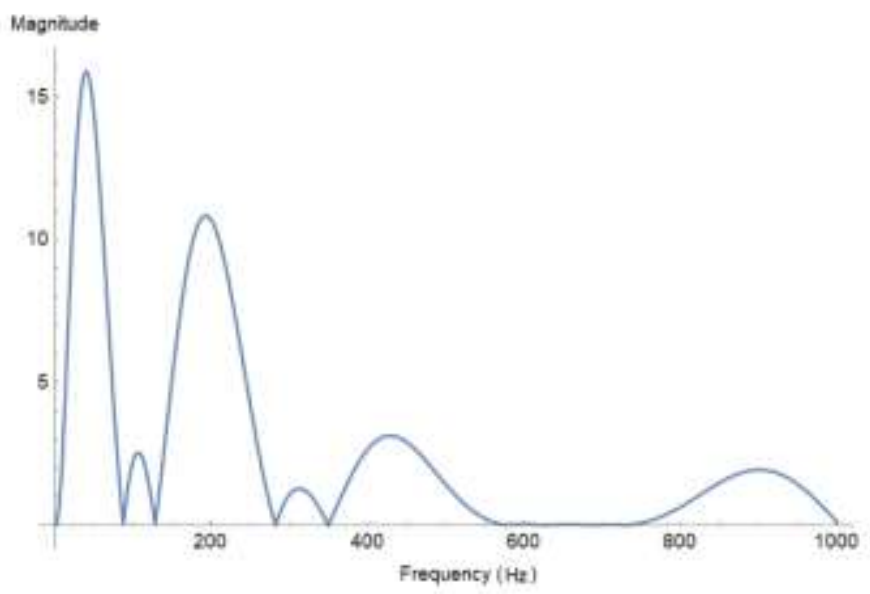

Figure 12: Characteristic polynomial

\section{Variation of Joint parameters and Comparison to Finite Element Model}

The Finite Element Model is created using Ansys Mechanical APDL, modelling the entire structure using solid elements, utilizing the material properties, dimensions and boundary conditions outlined in 3.3. The blocking mass will be represented as a solid cuboidal element with an initial width as $25 \mathrm{~mm}$,

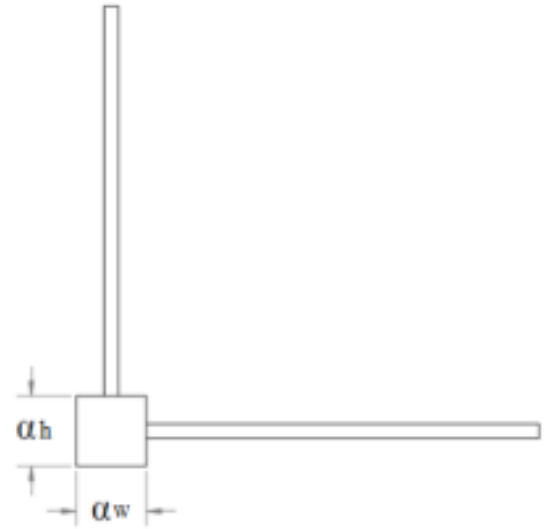

Figure 13: L-Beam structure with blocking mass

\subsection{Manipulation of Joint Dimensions}

For the purpose of simplicity, the dimensions of the joint will be scaled horizontally and vertically by a scaling factor, $\alpha$, ranging from 1-5 times the initial dimensions shown by Fig. 6 .

Table 2: Variation of natural frequencies due to scaling factor, $\alpha$

\begin{tabular}{|c|c|c|c|c|c|c|c|c|c|c|}
\hline Mode number & \multicolumn{8}{|c|}{ Natural Frequency (Hz) } \\
\hline & \multicolumn{2}{|c|}{$\alpha=1$} & \multicolumn{2}{|c|}{$\alpha=2$} & \multicolumn{2}{c|}{$\alpha=3$} & \multicolumn{2}{c|}{$\alpha=4$} & \multicolumn{2}{c|}{$\alpha=5$} \\
\hline & Wave & Ansys & Wave & Ansys & Wave & Ansys & Wave & Ansys & Wave & Ansys \\
\hline 1 & 87.741 & 87.066 & 86.499 & 84.921 & 84.638 & 82.596 & 82.111 & 79.593 & 80.117 & 75.154 \\
\hline 2 & 128.58 & 126.99 & 126.15 & 127.64 & 122.5 & 127.74 & 116.91 & 127.71 & 108.84 & 127.78 \\
\hline 3 & 282.48 & 280.49 & 272.47 & 273.38 & 238.78 & 264.13 & 189.16 & 248.41 & 154.74 & 222.41 \\
\hline
\end{tabular}




\begin{tabular}{|l|c|c|c|c|c|c|c|c|c|c|}
\hline 4 & 349.16 & 346.95 & 317.93 & 348.37 & 240.02 & 348.33 & 190.47 & 347.47 & 161.44 & 344.82 \\
\hline 5 & 576.5 & 579.59 & 380.14 & 564.19 & 293.68 & 539.72 & 277.14 & 493.41 & 268.46 & 417.43 \\
\hline
\end{tabular}

From Table 2, the wave model holds under two conditions; for lower frequencies and for a low scaling factor, $\alpha$ (and subsequently the size of the blocking mass), due to the joint being modelled as a perfectly rigid member.

Figure 7 gives insight into how the wave components for both reflection and transmission matrices across the joint transition and convert as the blocking mass size increases. The bending-longitudinal \& and longitudinal-bending wave components are dominated by a large transmission factor across the joint (large portion of the energy incident to the joint from beam 1 is then transferred to beam 2), except for the longitudinal onto longitudinal which is totally reflected (energy incident from beam 1 is reflected back onto beam 1).
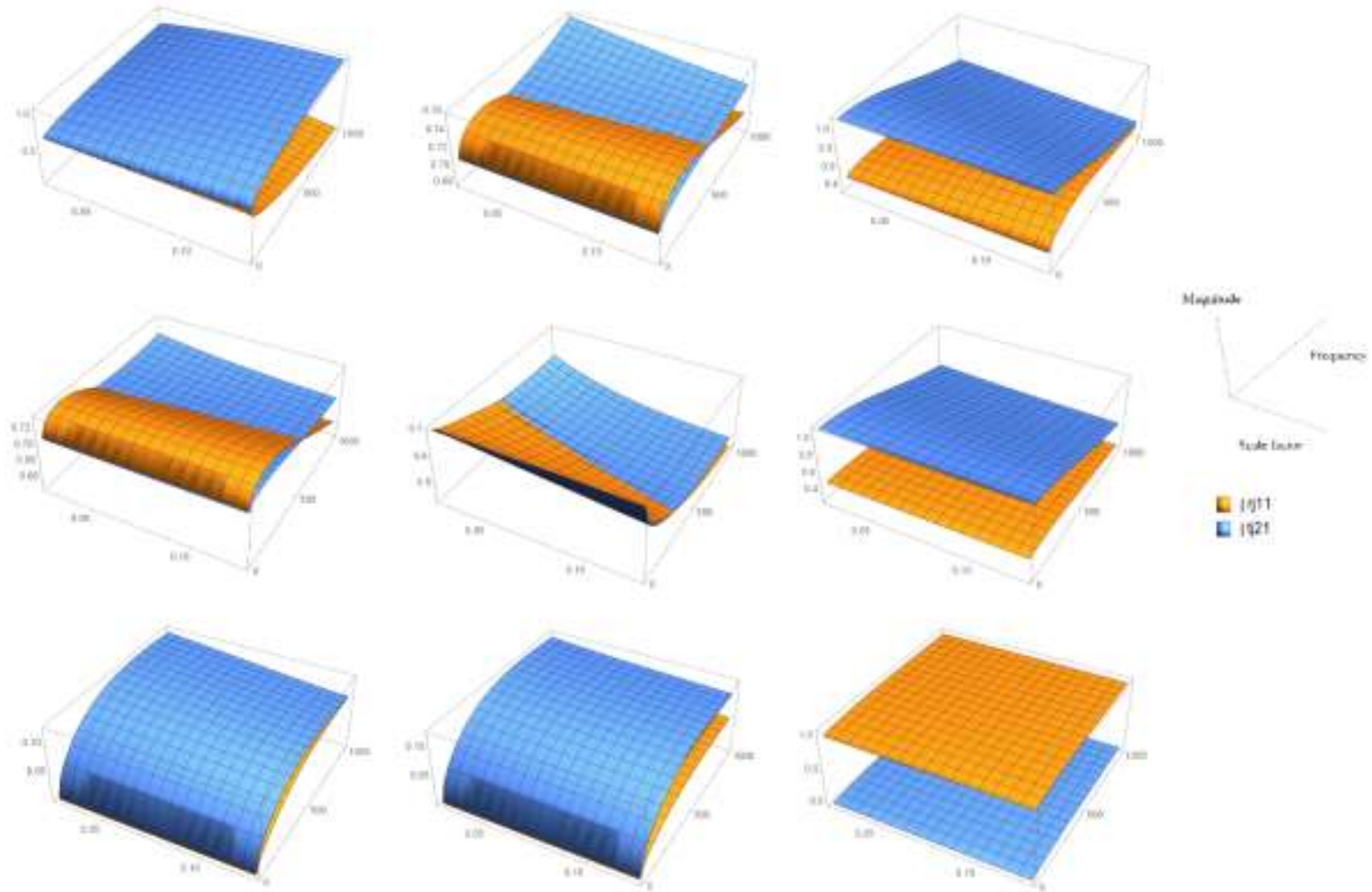

Figure 14: Parametric surface plots for elements of joint reflection (orange) and transmission (blue) matrices from $0-1000 \mathrm{~Hz}$ 

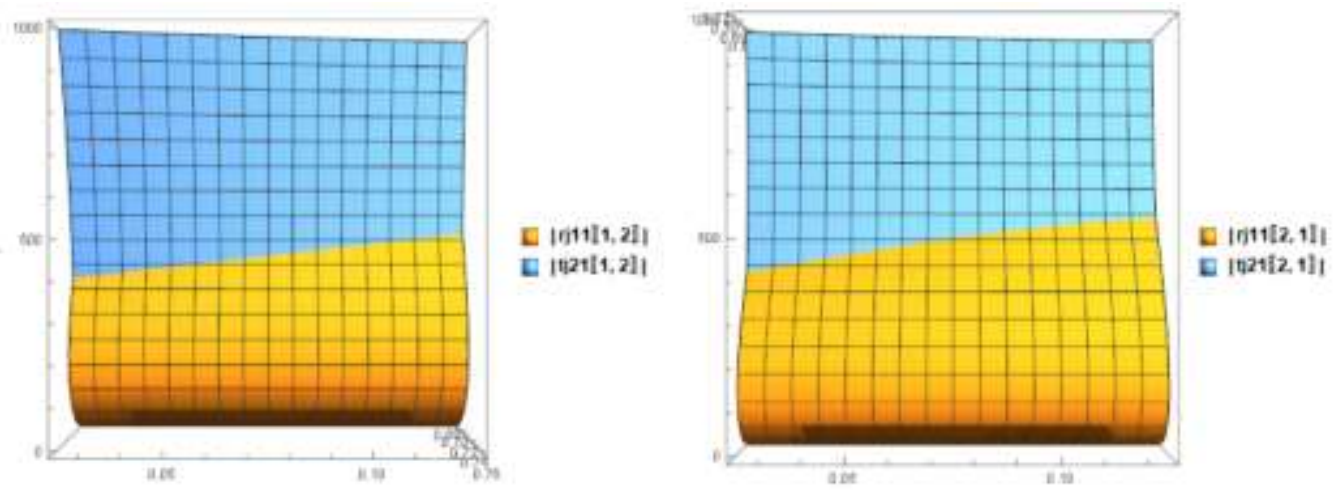

Figure 15: Transition frequency for wave component conversion of the $\mathrm{y}$ and $\mathrm{u}$ directions from 0 - 1000 $\mathrm{Hz}$

The wave components for the $\mathrm{y}$ and $\mathrm{u}$ direction, when converting to one another exhibit a transition frequency where the transmission (blue surface) supersedes the magnitude of the reflection components (orange surface), this transition frequency increases as the scaling factor, $\alpha$ also increases as seen in Fig. 8. Initially, for relatively low frequencies; a majority of the wave incident to beam 1 is reflected onto itself and as it approaches the transition frequency the reflected energy decreases, and the transmitted energy begins to increase beyond the magnitude of the respective reflection wave component.
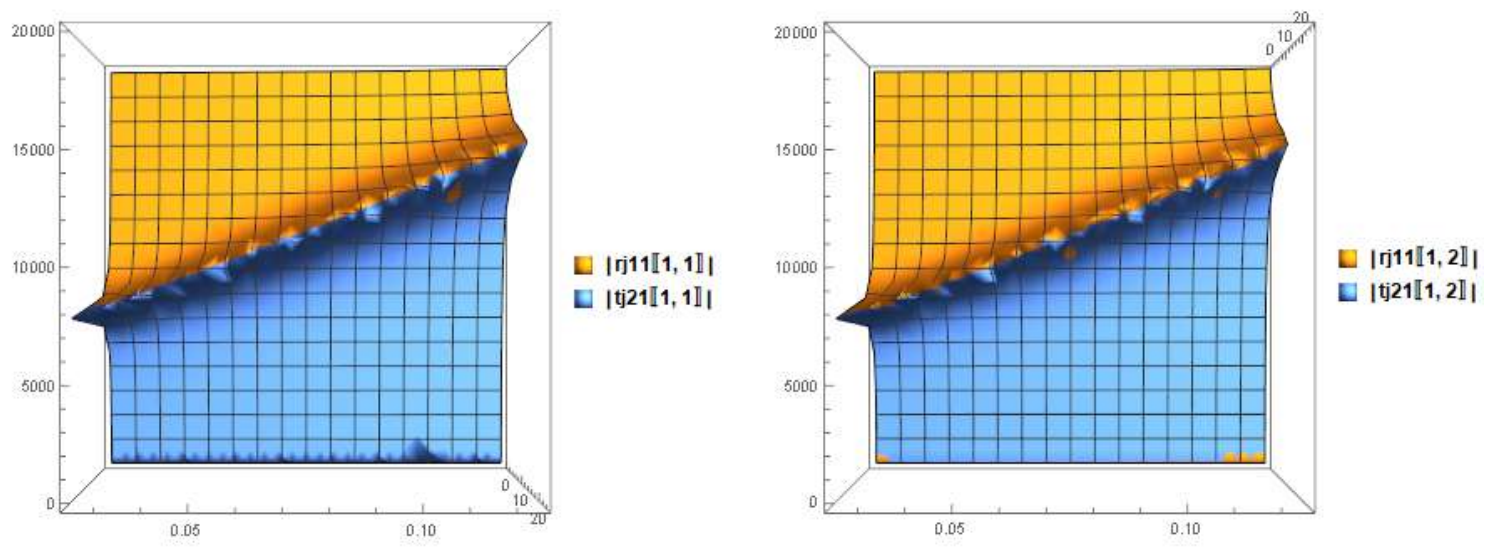

Figure 16: Secondary transition frequency shift from $0-20000 \mathrm{~Hz}$

Additionally, at higher frequencies there exists a secondary transition frequency dependent on $\alpha$ shown in Fig. 9 for the bending wave components in both the reflection and transmission matrices, $r_{j}$ and $t_{j}$. After this juncture the dominant wave component in terms of magnitude switches from the $t_{j}$ to $r_{j}$, described by Cremer, Heckl and Petersson [14] as the total reflection effect where the transmission factor vanishes completely leaving only the reflected portion, thus acting as a low pass filter.

\subsection{Manipulation of Joint Material}

The change of material will affect the mass of the joint and in turn the relative stiffness of the joint, increasing. Table 2 lists the natural frequency of the system described in 3.1 where the material of the joint is varied using physical properties for: Steel, Aluminium, Brass and Cast Iron, obtained from [26]. 
Table 3: Variation of Natural frequencies due to change of joint material

\begin{tabular}{|c|c|c|c|c|c|c|c|c|}
\hline $\begin{array}{c}\text { Mode } \\
\text { number }\end{array}$ & \multicolumn{9}{|c|}{ Natural Frequency (Hz) } \\
\hline & \multicolumn{2}{|c|}{ Aluminium } & \multicolumn{2}{c|}{ Cast Iron } & Low Carbon Steel & \multicolumn{2}{c|}{ Brass } \\
\hline & Wave & Ansys & Wave & Ansys & Wave & Ansys & Wave & Ansys \\
\hline & 87.741 & 87.064 & 87.741 & 87.066 & 87.741 & 87.066 & 87.741 & 87.064 \\
\hline 2 & 128.58 & 124.25 & 128.58 & 126.64 & 128.58 & 126.99 & 127.97 & 125.54 \\
\hline 3 & 283.15 & 280.46 & 282.48 & 280.49 & 282.48 & 280.49 & 282.48 & 280.46 \\
\hline 4 & 352.83 & 340.6 & 349.77 & 346.11 & 349.16 & 346.95 & 349.16 & 343.53 \\
\hline 5 & 587.15 & 579.5 & 579.16 & 579.59 & 576.5 & 579.59 & 574.5 & 579.49 \\
\hline
\end{tabular}

For the Wave and Ansys model, both showed little variation of the natural frequencies as the material was changed due to the relative size of the joint and thereby its mass, thus having relatively low weighting for determining the natural frequency. As with the results of manipulating the joint dimensions, the wave model only holds for lower frequencies and shows fair agreement across all materials chosen as shown in Table 3. Here the material of the joint was altered for each experiment, from a low density (aluminium) to a high density (brass), increasing the mass of the joint. For the wave model, as the mass of the joint increases the natural frequencies for each respective mode decreases. However, there becomes a disparity between the results of the wave and Ansys model for each of the materials as the mode number increases. This again can be attributed to the rigidness of the joint in the wave model, as it only includes the mass and the physical dimensions of the joint, thus the flexibility is not represented.

\section{Conclusion}

Using the wave vibration approach; the wave relations, reflection, transmission and propagation matrices and two methods of assembling and subsequently solving an L-beam structure were outlined in this paper. Fair agreement of in-plane natural frequencies between the wave and finite element model for the L- beam structure was achieved, however as the scaling factor, $\alpha$ increased, the agreement was not upheld. This shows that the wave model is limited to slender members as the flexibility cannot be appropriately represented as the model stands. From the surface plots of the reflection and transmission matrices, it is easily recognisable that by increasing the size of blocking mass the structure can be tuned to reflect higher frequencies and isolate vibration to a specific local of the structure. 


\section{References}

[1] Abed, Seraj Yousef. 2008. "Improving Productivity in Food Processing Industries Using Simulation - A Case Study." 12th WSEAS International Conference on Systems, Heraklion, Greece, July 23 - 25, 2008.

[2] Adam, Guido AO, and Detmar Zimmer. 2015. "On design for additive manufacturing: evaluating geometrical limitations." Rapid Prototyping Journal 21 (6):662-670.

[3] Advanced, Production Systems. 2018. Injection Molding Cost. Lyndon, Kentucky: Advanced Production Systems.

[4] Afrose, Mst Faujiya, SH Masood, Pio Iovenitti, Mostafa Nikzad, and Igor Sbarski. 2016. "Effects of part build orientations on fatigue behaviour of FDM-processed PLA material." Progress in Additive Manufacturing 1 (1-2):21-28.

[5] Ahn, Sung-Hoon, Michael Montero, Dan Odell, Shad Roundy, and Paul K Wright. 2002. "Anisotropic material properties of fused deposition modeling ABS." Rapid prototyping journal 8 (4):248-257.

[6] Bae, Ki-Hwan G., Lee A. Evans, and Alan Summers. 2016. "Lean Design and Analysis of a MilkRun Delivery System: Case Study." 2016 Winter Simulation Conference, Washington, DC, USA, December 11-14, 2016.

[7] Balderrama-Armendariz, Cesar Omar, Eric MacDonald, David Espalin, David Cortes-Saenz, Ryan Wicker, and Aide Maldonado-Macias. 2018. "Torsion analysis of the anisotropic behavior of FDM technology." The International Journal of Advanced Manufacturing Technology:1-11.

[8] Barbagallo, Mathias. 2013. "Statistical energy analysis and variational principles for the prediction of sound transmission in multilayered structures." KTH Royal Institute of Technology.

[9] Bezerra, Marcos Almeida, Ricardo Erthal Santelli, Eliane Padua Oliveira, Leonardo Silveira Villar, and Luciane Amélia Escaleira. 2008. "Response surface methodology (RSM) as a tool for optimization in analytical chemistry." Talanta 76 (5):965-977.

[10] Britannica, Encyclopædia. 2019. "Acrylonitrile-butadiene-styrene copolymer." Encyclopædia Britannica, inc., Last Modified 20/03/2019, accessed 16/05/2019. https://www.britannica.com/science/acrylonitrile-butadiene-styrene-copolymer.

[11] Chen, Wen-Chin, Gong-Loung Fu, Pei-Hao Tai, and Wei-Jaw Deng. 2009. "Process Parameter Optimisation for MIMO Plastic Injeciton Molding via Soft Computing." Elsevier 36 (2):8. doi: 10.1016/j.eswa.2007.10.020.

[12] Chen, Wen-Chin, Manh-Hung Ngyuyen, Wen-Hsin Chiu, Te-Ning Chen, and Pei-Hao Tai. 2016. "Optimization of the plastic injection molding process using the Taguchi method, RSM, and hybrid GA-PSO." The International Journal of Advanced Manufacturing Technology 83 (912):13. doi: 10.1007/s00170-015-7683-0.

[13] Chohan, Jasgurpreet Singh, and Rupinder Singh. 2017. "Pre and post processing techniques to improve surface characteristics of FDM parts: a state of art review and future applications." Rapid Prototyping Journal 23 (3):495-513.

[14] Cremer, Lothar, Manfred Heckl, BAT Petersson, and Paul J Remington. 2005. "Structure-borne sound: Structural vibrations and sound radiation at audio frequencies." Acoustical Society of America Journal 118:2754-2754.

[15] Dang, Xuan-Phuong. 2014. "General Frameworks for Optimization of Plastic Injection Molding Process Parameters." Elsevier 41:12. doi: 10.1016/j.simpat.2013.11.003.

[16] Di Angelo, Luca, and Paolo Di Stefano. 2011. "A neural network-based build time estimator for layer manufactured objects." The International Journal of Advanced Manufacturing Technology $57(1-4): 215-224$.

[17] Durgun, Ismail, and Rukiye Ertan. 2014. "Experimental investigation of FDM process for improvement of mechanical properties and production cost." Rapid Prototyping Journal 20 (3):228-235. 
[18] Faisal, KP, Falah Ummer, Hareesh K C, Munavir Ayaniyat, Nijab K, Nikesh P, and Jibi R. 2015. "Analysis and Simulation of Crankshaft Manufacturing Unit Using Arena." International Journal of Scientific and Research Publications 5 (2):7.

[19] Glassdoor. 2013. "Technician Salaries in Trinidad and Tobago." [Database for Company Reviews and Ratings], Last Modified December 22, 2013, accessed February 23. https://www.glassdoor.com/Salaries/trinidad-and-tobago-technician-salarySRCH_IL.0,19_IN227_KO20,30.htm.

[20] Graff, Karl F. 2012. Wave motion in elastic solids: Courier Corporation.

[21] Groover, Mikell P. 2007. Automation, Production Systems, and Computer-Integrated Manufacturing. 3rd ed. New Jersey, United States: Prentice Hall; 3 edition.

[22] Hashemi, Hosein, Massoud Kaykhaii, Ahmad Jamali Keikha, Zahra Sajjadi, and Majid Mirmoghaddam. 2019. "Application of response surface methodology for silver nano-particle stir bar sorptive extraction of heavy metals from drinking water samples: a Box-Behnken design." Analyst.

[23] Heshmat, M., M. A. El-Sharief, and M. G. El-Sebaie. 2013. "Simulation Modeling of Production Lines: A Case Study of Cement Production Line." Journal of Engineering Sciences 41 (3):8.

[24] Huttmeir, Andreas, Suzanne de Treville, Ann van Ackere, Leonard Monnier, and Johann Prenninger. 2009. "Trading Off Between Heijunka and Just-In-Sequence " International Journal of Production Economics 118 (2):7. doi: 10.1016/j.ijpe.2008.12.014.

[25] Jacobson, Jessica. 2015. "A Tale of Two Lines: Plastic Bottle Manufacturing Addresses Flexible, Dedicated Lines." Beverage Industry 107:2.

[26] Khurmi, RS, and JK Gupta. 2005. A textbook of machine design: Eurasia.

[27] Kumar, Vimal, Pratima Verma, Onkar, Suraj Pratap Singh, and Jitendra Katiyar. 2016. "Facility and Process Layout Analysis of an SME using Simulation: A Case Study of a Manufacturing Company." 2016 International Conference of Industrial Engineering and Operations Management, Kuala Lumpur, Malaysia, March 8-10, 2016.

[28] Lee, CS, SG Kim, HJ Kim, and SH Ahn. 2007. "Measurement of anisotropic compressive strength of rapid prototyping parts." Journal of materials processing technology 187:627-630.

[29] Mace, BR. 1984. "Wave reflection and transmission in beams." Journal of sound and vibration 97 (2):237-246.

[30] Matzka, Judith, Maria Di Mascolo, and Kai Furmans. 2012. "Buffer Sizing of a Heijunka Kanban System." Journal of Intelligent Manufacturing 23 (1):11. doi: 10.1007/s10845-009-0317-3.

[31] Mei, C. 2008. "Wave analysis of in-plane vibrations of H-and T-shaped planar frame structures." Journal of Vibration and Acoustics 130 (6):061004.

[32] Mei, C. 2012. "Wave analysis of in-plane vibrations of L-shaped and portal planar frame structures." Journal of Vibration and Acoustics 134 (2):021011.

[33] Mei, C, and BR Mace. 2004. "Wave reflection and transmission in Timoshenko beams and wave analysis of Timoshenko beam structures."

[34] Mohamed, Omar Ahmed, Syed Hasan Masood, and Jahar Lal Bhowmik. 2016. "Mathematical modeling and FDM process parameters optimization using response surface methodology based on Q-optimal design." Applied Mathematical Modelling 40 (23-24):10052-10073.

[35] Mohamed, Omar Ahmed, Syed Hasan Masood, and Jahar Lal Bhowmik. 2017a. "Experimental investigation for dynamic stiffness and dimensional accuracy of FDM manufactured part using IV-Optimal response surface design." Rapid Prototyping Journal 23 (4):736-749.

[36] Mohamed, Omar Ahmed, Syed Hasan Masood, and Jahar Lal Bhowmik. 2017b. "Influence of processing parameters on creep and recovery behavior of FDM manufactured part using definitive screening design and ANN." Rapid Prototyping Journal 23 (6):998-1010. 
[37] Mohammed, Abdullah. 2017. "Don't Just Teach a Man to Fish: Are Our Caribbean SME Policies Fundamentally Wrong? ." Technology news from T\&T, Last Modified December 11, 2017, accessed January 7. https://technewstt.com/sme-policies/.

[38] Montgomery, Douglas C. 2017. Design and analysis of experiments: John wiley \& sons.

[39] NOGUCHI, Hiroshi, and Kazuhiro UCHIDA. 2000. "FEM Analysis of Hybrid Structural Frames with R/C Columns and Steel Beams." XIAO Yun. Proceedings of 6th ASCCS conference.

[40] Rayegani, Farzad, and Godfrey C Onwubolu. 2014. "Fused deposition modelling (FDM) process parameter prediction and optimization using group method for data handling (GMDH) and differential evolution (DE)." The International Journal of Advanced Manufacturing Technology 73 (1-4):509-519.

[41] Russell, Suzana N., and Harvey H. Millar. 2014. "Competitive Priorities of Manufacturing Firms in the Caribbean." Journal of Business and Management 16 (10):10. doi: 10.9790/487X161017282.

[42] Rymaszewska, Anna Dorota. 2014. "The Challenges of Lean Manufacturing Implementation in SMEs." Benchmarking an International Journal 21 (6):15. doi: 10.1108/BIJ-10-2012-0065.

[43] Sood, Anoop K, Raj K Ohdar, and Siba S Mahapatra. 2012. "Experimental investigation and empirical modelling of FDM process for compressive strength improvement." Journal of Advanced Research 3 (1):81-90.

[44] Sood, Anoop Kumar. 2011. "Study on parametric optimization of fused deposition modelling (FDM) process."

[45] Sood, Anoop Kumar, Raj K Ohdar, and Siba S Mahapatra. 2010. "Parametric appraisal of mechanical property of fused deposition modelling processed parts." Materials \& Design 31 (1):287-295.

[46] Sundar, R., A. N. Balaji, and R. M. Satheesh Kumar. 2014. "A Review on Lean Manufacturing Implementation Techniques." 12th Global Congress on Manufacturing and Management, GCMM 2014, Vellore, India, 30 December, 2014.

[47] Tatar, İlhan. 2013. "Vibration characteristics of portal frames." Izmir Institute of Technology.

[48] Toyota, ATLAS. 2018. Do You Know Your Average Forklift Cost per Hour? Illinois, United States of America: Atlas Toyota.

[49] Ullah, M., A. M. Khan, R. Nawaz, and R. Akhtar. 2016. "Process Improvement for PET Bottles Manufacturing Company Using Six Sigma Approach." Technical Journal 21 (11):9.

[50] Wen, Hua Bing, Lin Bo Liu, Zi Long Peng, and Qi Dong Zhong. 2014. "Research on the effects of Blocking Mass on Vibration Resistance Performance of L-shaped plates." Applied Mechanics and Materials.

[51] Womack, James P., and Daniel T. Jones. 2003. Lean Thinking: Banish Waste and Create Wealth in Your Corporation. 2 ed. Michigan, United States of America: Free Press.

[52] Woodhouse, J. 1981. "An approach to the theoretical background of statistical energy analysis applied to structural vibration." The Journal of the Acoustical Society of America 69 (6):16951709.

[53] Xu, Yingjie, QingWen Zhang, Weihong Zhang, and Pan Zhang. 2015. "Optimization of Injection Molding Process Parameters to Improve the Mechanical Performance of Polymer Product Against Impact." The International Journal of Advanced Manufacturing Technology 76 (9-12):9. doi: 10.1007/s00170-014-6434-y.

[54] Zhou, Xuandao, Yun Zhang, Ting Mao, and Huamin Zhou. 2017. "Monitoring and Dynamic Control of Quality Stability for Injection Molding Process." Journal of Materials Processing Technology 249:8. doi: 10.1016/j.jmatprotec.2017.05.038.

[55] Ziemian, Constance, Mala Sharma, and Sophia Ziemian. 2012. "Anisotropic mechanical properties of ABS parts fabricated by fused deposition modelling." In Mechanical engineering. InTech. 